\title{
Nowcasting of the surface layer of turbulence at Paranal Observatory
}

\author{
Timothy Butterley ${ }^{\mathrm{a}}$, James Osborn ${ }^{\mathrm{a}}$, Marc Sarazin ${ }^{\mathrm{b}}$, and Richard Wilson ${ }^{\mathrm{a}}$ \\ ${ }^{a}$ Centre for Advanced Instrumentation, Department of Physics, University of Durham, South \\ Road, Durham, DH1 3LE, UK \\ ${ }^{\text {b} E u r o p e a n ~ S o u t h e r n ~ O b s e r v a t o r y ~(E S O), ~ K a r l-S c h w a r z s c h i l d-S t r . ~ 2, ~ D-85748 ~ G a r c h i n g, ~}$
} Germany

\begin{abstract}
The surface layer of turbulence at Paranal Observatory is monitored by an automated surface layer Slope Detection and Ranging (SLODAR) profiler. This has two main purposes. Firstly, the high resolution surface layer profile it provides allows the fraction of turbulence that is below the level of the Unit Telescope (UT) mirrors to be estimated, and thus a total seeing estimate for the UTs to be made. Secondly, it can measure up to the minimum altitude at which the MASS is sensitive, so together they provide a profile of the full atmosphere. This allows performance of the Adaptive Optics Facility (AOF) to be predicted before committing the instrument to an observation.

The SLODAR instrument has been operating at Paranal since August 2013. We present an overview of the SLODAR data collected to date and concurrent meteorological measurements made on-site, and place bounds on the accuracy with which the surface layer turbulence can be "nowcasted" based on live local weather data. This could be an invaluable tool in scheduling adaptive optics observations.
\end{abstract}

Keywords: SLODAR, turbulence profiling

\section{INTRODUCTION}

Paranal Observatory has a facility Slope Detection and Ranging (SLODAR) ${ }^{1,2}$ instrument that was built for the European Southern Observatory (ESO) by Durham University. The SLODAR instrument is part of the astronomical site monitor (ASM), a suite of instruments that constantly monitors the ambient conditions at the observatory site. The SLODAR provides surface layer and ground layer profiling to support the Adaptive Optics Facility (AOF).

We describe a method for predicting, or "nowcasting", the current surface layer turbulence strength at the observatory based on measurements of the current local meteorological conditions. This has a number of potential applications:

- In the event of a failure of the SLODAR instrument, an estimate of the surface layer strength could still be provided.

- When the SLODAR is operating in a mode with coarse vertical resolution and the surface layer is entirely in the first resolution element (see section 2 for more information) the profile could be supplemented with an estimate of the extent of the surface layer above the ground.

- The method could be extended by incorporating information from weather forecasts so the future surface layer strength could be forecasted.

Further author information: (Send correspondence to T.B.)

T.B.: E-mail: timothy butterley@durham.ac.uk, Telephone: +44 1913343714 
- The same technique could be applied at other observatory sites. At sites where it is not feasible to install a permanent surface layer monitor, such an instrument could be installed temporarily and operated in campaigns. The campaign data could be used, together with this method, to continue to provide estimates of the strength of the surface layer.

The paper is structured as follows: section 2 describes the Paranal surface layer SLODAR instrument and its capabilities; section 3 describes the meteorological parameters available from the ASM; section 4 describes the surface layer nowcasting method and results and section 5 contains the conclusions.

\section{THE SLODAR INSTRUMENT}

The robotic SLODAR instrument (shown in figure 1), installed at Paranal in 2013, consists of a pair of $8 \times 8$ subaperture Shack-Hartmann wavefront sensors that can observe pairs of stars with separations ranging from 2 arcmin to 12 arcmin. This yields 8-layer turbulence profiles up to a maximum altitude of approximately $500 \mathrm{~m}$. Narrow targets profile higher but with coarser resolution - see figure 2. Figure 3 shows some example profile data for one night in April 2014.

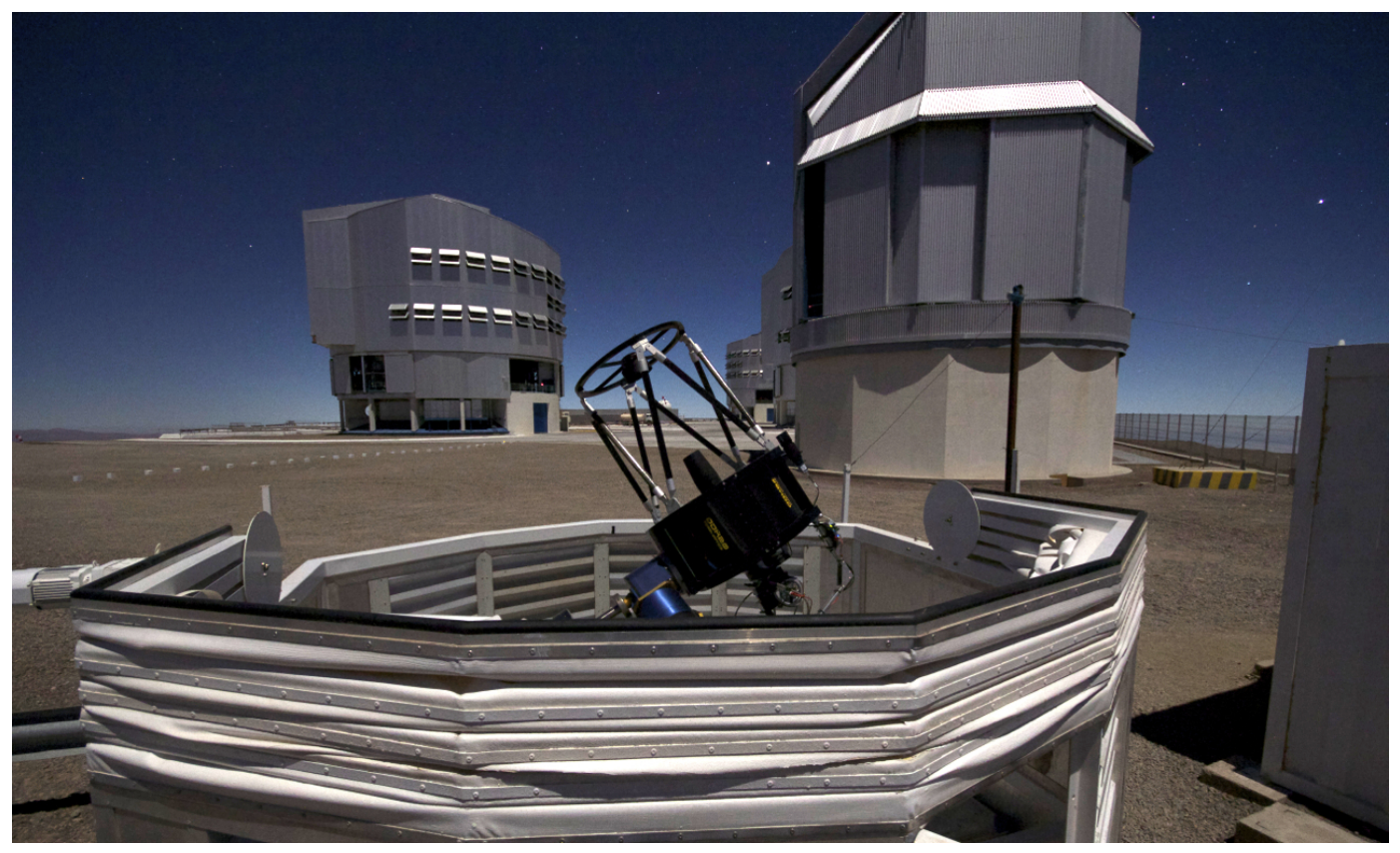

Figure 1. The Paranal robotic SLODAR instrument (foreground) with the VST (on the right) and UTs (left and behind the VST) in the background.

In general, the surface layer of turbulence is not resolved by the SLODAR i.e. all or most of it is observed within the first resolution element of the instrument so the thickness of the surface layer can not be determined. The prototype surface-layer SLODAR used wider targets $(12.5-15.5$ arcmin) so was better able to resolve the surface layer. In order to provide a better estimate of the surface layer contribution below the Unit Telescope (UT) dome height, when the surface layer is not resolved it is assumed to follow an exponential model with a scale height of $5 \mathrm{~m}$. Figure 4 shows the expected median seeing as a function of height. ${ }^{3}$

\section{METEOROLOGICAL DATA}

Meteorological parameters at Paranal are measured by sensors on a mast close to the SLODAR. The following parameters are recorded every minute:

- Temperature (30 m above ground, 10m above ground, ground level and $20 \mathrm{~m}$ below platform). 


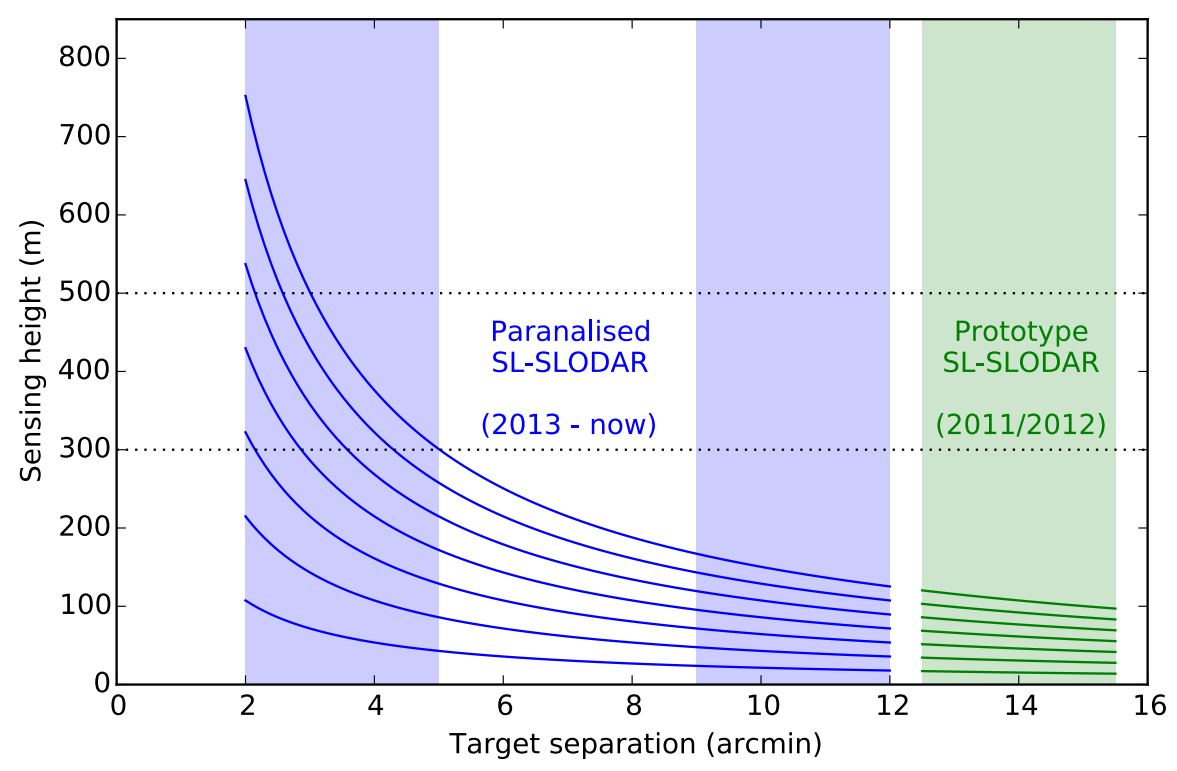

Figure 2. Heights of the 8 fitted SLODAR layers as a function of target star separation for a target at zenith. The first layer is always at $0 \mathrm{~m}$.

- Wind speed and direction (30 $\mathrm{m}$ and $10 \mathrm{~m}$ above the ground).

- Relative humidity (30 $\mathrm{m}$ and $10 \mathrm{~m}$ above the ground).

- Air pressure.

\section{NOWCASTING THE SURFACE LAYER}

Prediction of high altitude turbulence requires meso-scale modelling ${ }^{4,5}$ but we can attempt to estimate the current surface layer strength based only on local meteorological parameters. A very simple artificial neural network (ANN) was trained to predict the current surface layer strength based on current meteorological parameters. The ANN in question is a multilayer perceptron with one hidden layer containing 12 neurons. This was implemented using the "Keras" library* for Python, with the "Theano" backend.

The meteorological parameters listed in section 3 constitute most of the inputs to the ANN. These paremeters were normalized such that each parameter had a mean of 0 and standard deviation of 1 when seen by the ANN. The following additional parameters were also included as inputs:

- Current rate of change of meteorological parameters (computed for each dataset from several consecutive measurements and also normalized).

- Time of year of each set of measurements, to aid the ANN in recognising seasonal variations. In order to encode the periodicity of this parameter it is expressed as sine/cosine pair of inputs, given by

$$
\begin{aligned}
& \tau_{x}=\cos \left(\frac{2 \pi t}{365.25 \times 24 \times 3600}\right) \\
& \tau_{y}=\sin \left(\frac{2 \pi t}{365.25 \times 24 \times 3600}\right)
\end{aligned}
$$

where $t$ is the time in seconds from the start of the year.

\footnotetext{
${ }^{*}$ https://keras.io
} 


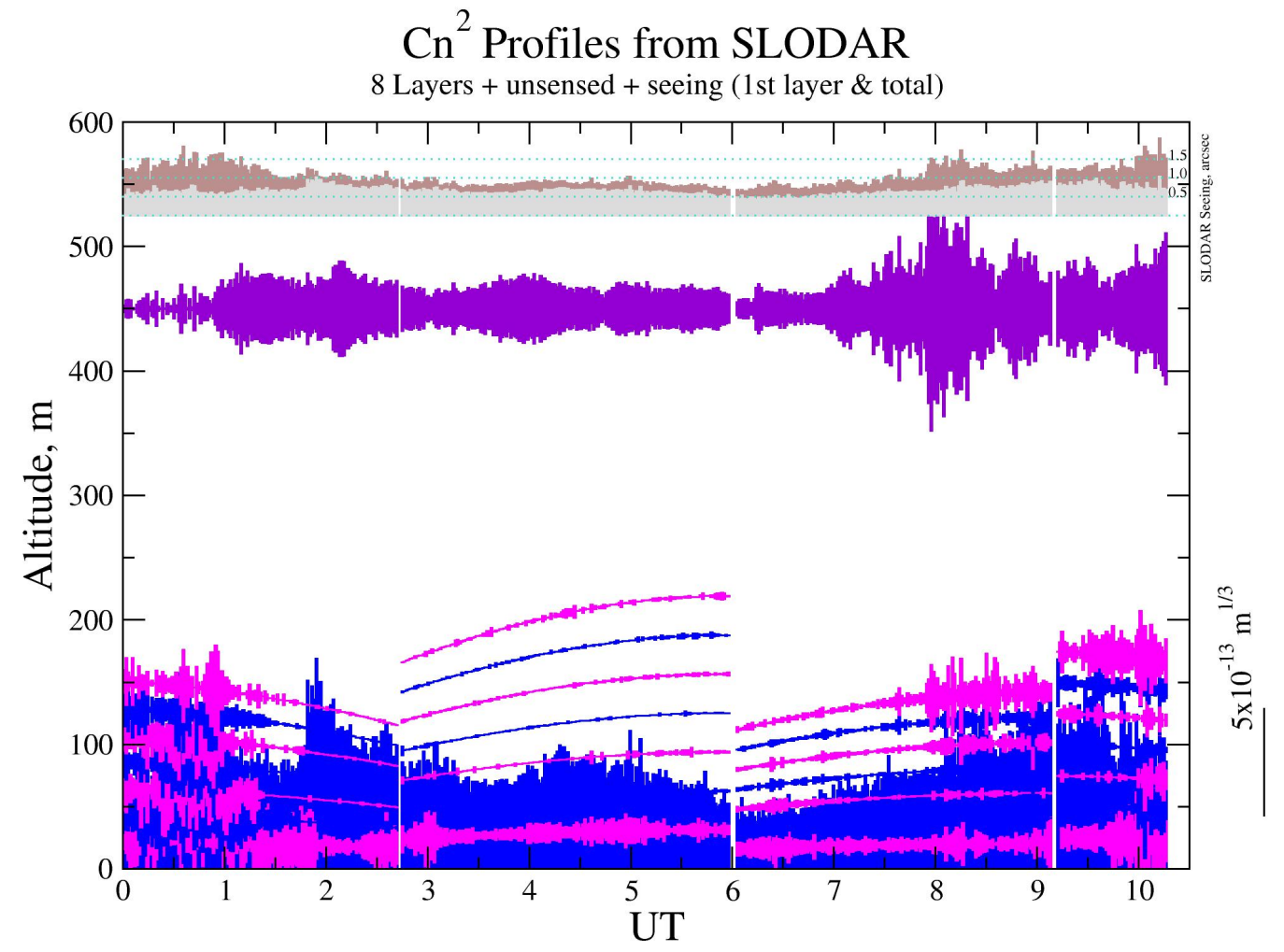

Figure 3. Example turbulence profile data (night starting 22 April 2014). The pink and blue traces represent the 8 resolution elements; each trace is centred at the height of the fitted layer and the thickness indicates the integrated $C_{n}^{2} d h$ in the layer. Note that the traces change in height depending on the target separation and zenith angle. The purple trace shows the total integrated $C_{n}^{2} d h$ above the maximum sensing height. The grey and brown traces show the seeing due to the ground layer and full atmosphere respectively.

- Time of day of each set of measurements, similarly expressed as a sine/cosine pair of inputs to encode 24-hour periodicity.

The sole output of the ANN is the surface layer strength integrated up to $30 \mathrm{~m}$, denoted by $J_{\text {ANN }}$. The upper limit of $30 \mathrm{~m}$ was chosen, somewhat arbitrarily, to match the maximum height of the meteorological sensors. Different surface layer parameters could be chosen as required for particular applications. The data set used to train the ANN consists of $80 \%$ of the nights of simultaneous SLODAR and meteorological data available up to March 2017, selected at random. The remaining $20 \%$ of nights were reserved as a test data set to assess the performance of the ANN. The measurements of surface layer strength up to $30 \mathrm{~m}$ are denoted by $J_{\text {SLODAR }}$.

Figure 5 shows a comparison between the measured surface layer strength and that predicted by the ANN. Agreement is by no means perfect but there is strong correlation between the predicted and measured surface layer strength, demonstrating the validity of the method. It is likely that the result could be improved further by refining the training process of the ANN.

\section{CONCLUSION}

The results here demonstrate that the current surface layer turbulence strength at Paranal can be "nowcasted" based solely on local meteorological measurements. The method could therefore be applied, at Paranal or at other observatories, as an alternative to a full time surface layer monitor. 


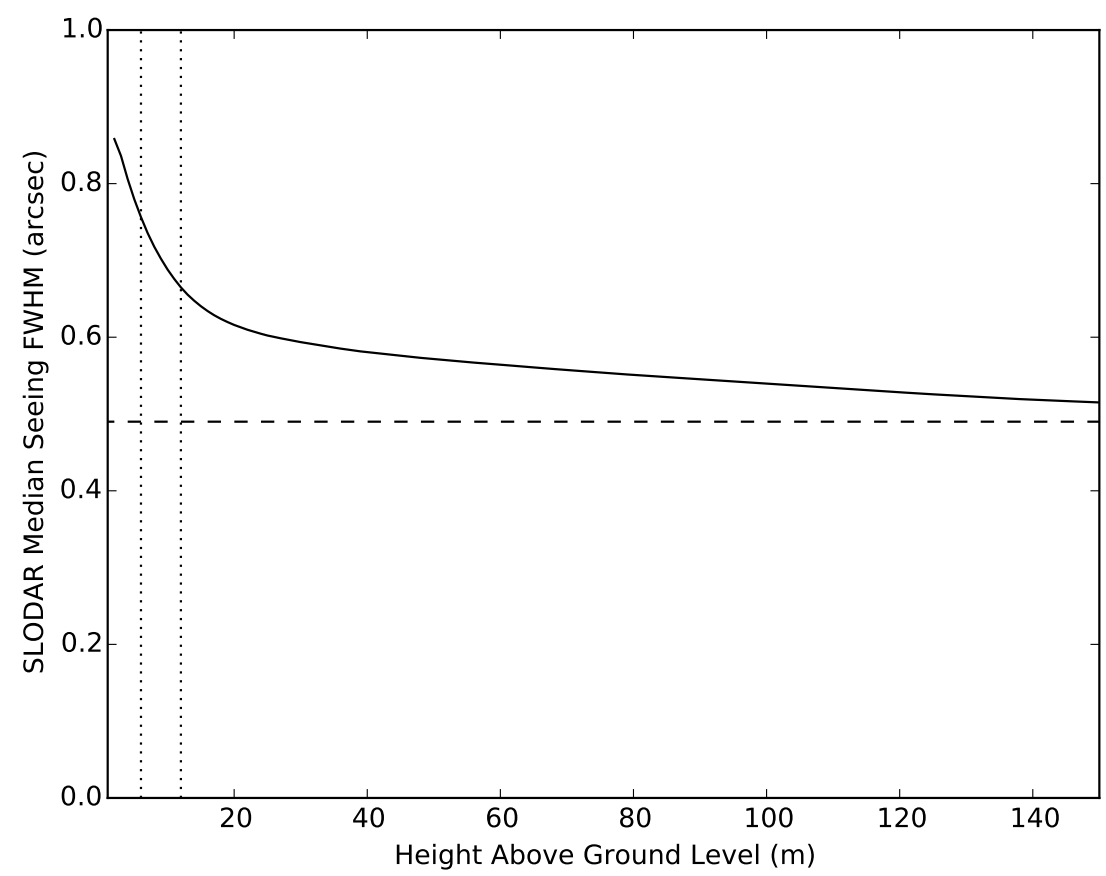

Figure 4. SLODAR median seeing as a function of telescope altitude. The horizontal broken line is the median seeing at $300 \mathrm{~m}$. The vertical dotted lines are at the heights of the DIMM and UT.

The method could be developed further by extending the ANN output to a wider range of surface layer parameters than just the $30 \mathrm{~m}$ integral, and including weather forecasts as additional inputs to the ANN and attempting to train it to forecast the future surface layer strength.

\section{ACKNOWLEDGMENTS}

TB, JO and RWW are grateful to the Science and Technology Facilities Committee (STFC) for financial support (grant reference ST/P000541/1). TB would like to thank Andrew Reeves for useful discussions about ANN.

\section{REFERENCES}

[1] Wilson, R. W., "SLODAR: measuring optical turbulence altitude with a Shack-Hartmann wavefront sensor," Mon. Not. R. Astron. Soc. 337, 103-108 (2002).

[2] Butterley, T., Wilson, R. W., and Sarazin, M., "Determination of the profile of atmospheric optical turbulence strength from SLODAR data," Mon. Not. R. Astron. Soc. 369, 835-845 (2006).

[3] Butterley, T., Wilson, R., and Sarazin, M., "Processing of external slodar turbulence profile measurements to support adaptive optics performance predictions," in [Adaptive Optics for Extremely Large Telescopes 4-Conference Proceedings], 1(1) (2015).

[4] Lascaux, F., Masciadri, E., and Fini, L., "Forecast of surface layer meteorological parameters at Cerro Paranal with a mesoscale atmospherical model," Mon. Not. R. Astron. Soc. 449, 1664-1678 (May 2015).

[5] Masciadri, E., Lascaux, F., Turchi, A., and Fini, L., "Optical turbulence forecast: ready for an operational application," Mon. Not. R. Astron. Soc. 466, 520-539 (Apr. 2017). 

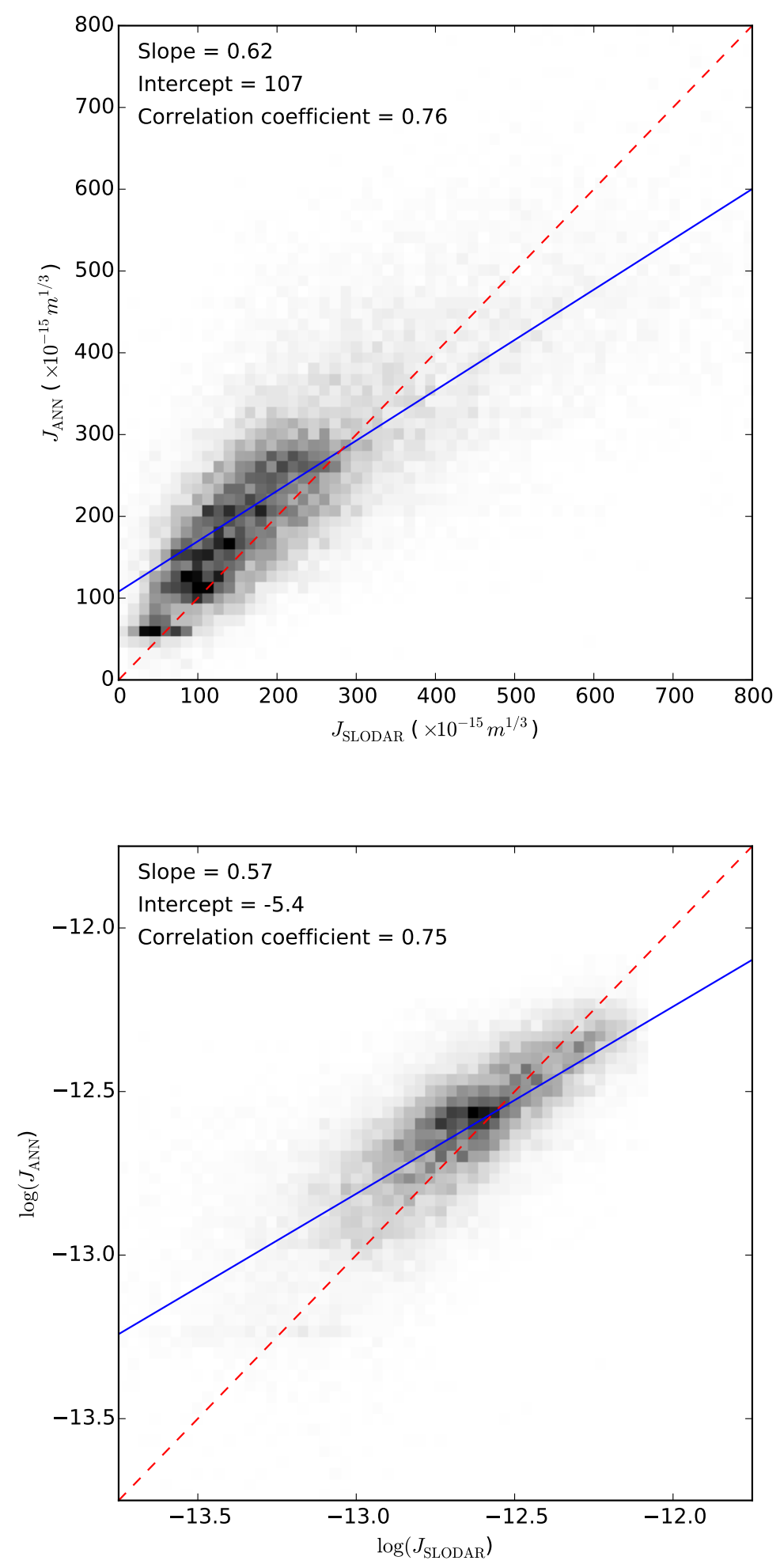

Figure 5. Measured vs ANN-predicted surface layer strength. Top: linear scale; bottom: $\log -\log$ scale. In each case the blue line shows a linear regression and the red broken line shows the "perfect agreement" case. 